\title{
Circulating Serum Copper Is Associated with Atherosclerotic Cardiovascular Disease, but Not Venous Thromboembolism: A Prospective Cohort Study
}

\author{
Setor K. Kunutsor ${ }^{a, b, c, d ~ R i c h a r d ~ S . ~ D e y ~}{ }^{\mathrm{e}}$ Jari A. Laukkanen ${ }^{c, f, g}$

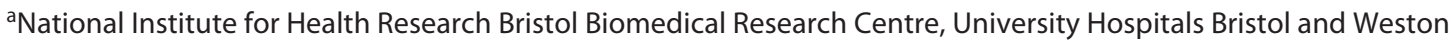 \\ NHS Foundation Trust and the University of Bristol, Bristol, UK; ${ }^{\text {b} T r a n s l a t i o n a l ~ H e a l t h ~ S c i e n c e s, ~ B r i s t o l ~ M e d i c a l ~}$ \\ School, University of Bristol, Learning \& Research, Building (Level 1), Southmead Hospital, Bristol, UK; 'Central \\ Finland Health Care District, Department of Medicine, Jyväskylä, Finland; dDiabetes Research Centre, University of

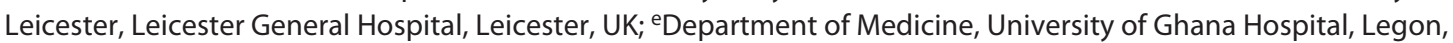 \\ Ghana; 'Institute of Clinical Medicine, Department of Medicine, University of Eastern Finland, Kuopio, Finland; \\ IInstitute of Public Health and Clinical Nutrition, University of Eastern Finland, Kuopio, Finland
}

\section{Keywords}

Serum copper - Venous thromboembolism - Atherosclerotic cardiovascular disease $\cdot$ Risk factor · Cohort study

\begin{abstract}
Background and Objective: Serum copper has been linked to the risk of atherosclerotic cardiovascular disease (CVD). However, the potential association between serum copper and venous thromboembolism (VTE) is not known. The principal aim was to evaluate the potential prospective association between serum copper and VTE risk. A secondary aim was to confirm or refute previously reported associations between serum copper and atherosclerotic CVD. Methods: Serum copper was measured at baseline using atomic absorption spectrometry in 2,492 men aged 42-61 years without a history of VTE in the Kuopio Ischemic Heart Disease prospective cohort study. Cox regression models were used to calculate hazard ratios (HRs) with 95\% confidence interval (CI) for VTE. Results: During a median follow-up of 27.0 years, 166 VTE events occurred. The risk of VTE per 1 standard deviation increase in serum copper in age-adjusted analysis was HR: 1.02; 95\% Cl: 0.88-1.20, which was attenuated to HR: 0.99; $95 \% \mathrm{Cl}: 0.82-1.19$, following further adjustment for several
\end{abstract}

established and emerging risk factors. Comparing the top versus bottom tertiles of serum copper, the corresponding adjusted HRs were 1.16 (95\% Cl: 0.80-1.66) and 1.11 (95\% Cl: 0.74-1.68), respectively. In 1,901 men without a history of coronary heart disease (CHD), the multivariable-adjusted $\mathrm{HR}$ for CHD was 1.32 (95\% Cl: 1.10-1.59) comparing extreme tertiles of serum copper. Conclusion: In middle-aged Finnish men, we confirmed previously reported associations between high serum copper levels and increased risk of atherosclerotic CVD, but serum copper was not associated with future VTE risk. Other large-scale prospective studies conducted in women, other age-groups, and other populations are needed to confirm or refute these findings.

(c) 2021 S. Karger AG, Basel

\section{Introduction}

Despite considerable advances in preventive and management strategies, cardiovascular disease (CVD) is still the leading cause of death globally [1]. Atherosclerotic CVDs, which include ischemic heart disease and cerebrovascular disease (mainly ischemic stroke) [1], are the major manifestations of CVD. Venous thromboembolism 
(VTE) (comprising deep vein thrombosis and pulmonary embolism) is a vascular condition that is closely related to atherosclerotic CVDs. They both share several common risk factors such as age, obesity, and cigarette smoking [2, 3] and mechanistic pathways such as coagulation, platelet activation, and dyslipidemia [4]. Just like atherosclerotic CVD, VTE constitutes a substantial public health burden and is associated with substantial morbidity, high economic costs, and premature mortality $[5,6]$.

Copper like other essential trace elements is an important cofactor of numerous enzymes and is involved in a number of cellular processes such as transcriptional regulation, oxidoreductases, inflammation, immune function, mitochondrial electron transport, and free radical scavenging [7]. Given that copper is involved in numerous biological processes, its insufficiency, deficiency, or toxic levels can lead to many disease states. The role of copper in the pathogenesis of CVD has been overlooked. The myocardium is one of the organs with the highest concentrations of copper, after the liver, brain, and kidney. Hence, the heart is one of the main organs affected by copper deficiency, which causes a reduction in metabolism and energy supply in the myocardium [8]. Serum copper deficiency has been linked with the risk of atherosclerotic CVD outcomes such as coronary heart disease (CHD) [9] via pathways such as hypercholesterolemia, hypertension, hyperuricemia, and impaired glucose tolerance $[9,10]$. It has been suggested that copper deficiency could be driving much of the current burden of atherosclerotic CVD in the population [9]. Conversely, because of the role of copper in catalyzing oxidation-reduction reactions, which play an important role in the pathogenesis of CHD, copper may also play a role in atherogenesis. High serum copper levels have been linked to an increased risk of atherosclerotic CVDs in a number of observational case-control [11] and cohort studies [12, 13]. Previously reported associations between serum copper and CVD outcomes have mostly been inconsistent.

Given the shared pathways between copper, atherosclerotic CVD, and VTE, we hypothesized that serum copper may be linked to the risk of VTE. To explore this hypothesis, our principal aim was to assess the nature and magnitude of the prospective association of serum circulating copper with risk of VTE, using a population-based prospective cohort of 2,492 middle-aged Finnish men. A secondary aim was to confirm or refute previously reported associations between serum copper and atherosclerotic CVD.

\section{Methods}

Reporting of the study conforms to broad EQUATOR guidelines [14] and was conducted according to STrengthening the Reporting of OBservational studies in Epidemiology guidelines for reporting observational studies in epidemiology (online suppl. material 1, available at www.karger.com/doi/10.1159/000519906). Written informed consent was obtained from all participants, the research protocol was approved by the institutional review board of the University of Eastern Finland (reference \#:143/97), and all study procedures were conducted according to the Declaration of Helsinki. The study population formed part of the ongoing Kuopio Ischemic Heart Disease population-based prospective cohort study, which was set up primarily to investigate established and emerging risk factors for atherosclerotic CVD and related outcomes in Eastern Finland. Participants were a randomly selected sample from a general population of Kuopio and the surrounding area in Eastern Finland. Study design, recruitment methods, and assessment of lifestyle factors, medical history, and blood-based markers have been described previously [15-22]. Of the 3,433 randomly selected men who participated in the baseline study conducted between March 1984 and December 1989, 3,235 were found to be eligible. Of this number, 186 did not respond to the invitation, 367 declined to give informed consent, and 2,682 volunteered to participate. The men were aged $42-61$ years at the time of the baseline examination. The current analysis is based on 2,492 men with nonmissing data on serum copper, relevant covariates, and first VTE events. A subsidiary analysis of the association between serum copper and risk of CHD was based on 1,901 men without a previous history of CHD and nonmissing data on serum copper, relevant covariates, and first CHD events.

Besides fasting overnight before blood collection, participants were told to abstain from drinking alcohol for at least 3 days and from smoking for at least $12 \mathrm{~h}$ before blood tests. Copper-free needles and tubes were used for collecting and storing blood. Serum copper concentrations were determined by atomic absorption spectrometry from frozen samples stored at $-20^{\circ} \mathrm{C}$ for $1-5$ years prior to analyses. All daily batches had control serum samples. The Perkin Elmer 306 atomic absorption spectrophotometer (Norwalk, CT, USA) was used for measurements, which involved the use of acetylene-air (1:4) flame technique with reference standards dissolved in 5\% glycerol. The between-batch coefficient of variation was $4.0 \%$. We included all incident VTE events that occurred from study entry to 2018. The events were identified by computer linkage to the National Hospital Discharge Registry Data maintained by the Finnish Institute for Health and Welfare, and their diagnoses required positive imaging tests. The medical documents for each potential VTE case were cross-checked in detail, and each event was validated by 2 physicians who were blinded to the exposures. The ICD 10 codes (I26, I80, and I82) were used to code and classify each VTE case. We included all first incident cases of CHD that occurred from study entry until the end of 2018. The diagnostic classification of $\mathrm{CHD}$ was based on symptoms, electrocardiographic findings, cardiac enzyme elevations, and autopsy outcomes [23].

\section{Statistical Analysis}

Variables with skewed distributions were natural log-transformed to achieve approximately symmetrical distributions. Descriptive data were presented as means (standard deviation, SD) or medians (interquartile range, IQR) reported for continuous variables and $n$ (percentages) for categorical variables. Partial correlation coef- 
Table 1. Baseline participant characteristics and correlates of serum copper $(N=2,492)$

\begin{tabular}{|c|c|c|c|}
\hline \multicolumn{4}{|l|}{ Questionnaire/prevalent conditions } \\
\hline Age at survey (yr) & $53(5)$ & $0.06(0.02,0.10)^{*}$ & $0.01(0.00,0.02)^{*}$ \\
\hline Alcohol consumption (g/wk) & $31.4(6.2-91.4)$ & $0.11(0.07,0.15)^{* * *}$ & $0.02(0.01,0.03)^{* * *}$ \\
\hline No & $2,390(95.9)$ & - & Ref \\
\hline Yes & $102(4.1)$ & - & $0.01(-0.03,0.05)$ \\
\hline \multicolumn{4}{|l|}{ Smoking status } \\
\hline Other & $1,706(68.5)$ & - & Ref \\
\hline Current & $786(31.5)$ & - & $0.06(0.05,0.08)^{* * *}$ \\
\hline No & $2,476(99.4)$ & - & Ref \\
\hline Yes & $16(0.6)$ & - & $0.07(-0.02,0.16)$ \\
\hline \multicolumn{4}{|l|}{ History of cancer } \\
\hline No & $2,450(98.3)$ & - & Ref \\
\hline Yes & $42(1.7)$ & - & $-0.06(-0.11,-0.00)^{*}$ \\
\hline \multicolumn{4}{|l|}{ Physical measurements } \\
\hline $\mathrm{BMI}\left(\mathrm{kg} / \mathrm{m}^{2}\right)$ & $26.9(3.6)$ & $0.09(0.05,0.13)^{* * *}$ & $0.02(0.01,0.02)^{* * *}$ \\
\hline $\mathrm{SBP}(\mathrm{mm} \mathrm{Hg})$ & $134(17)$ & $0.09(0.05,0.13)^{* * *}$ & $0.02(0.01,0.02)^{* * *}$ \\
\hline $\mathrm{DBP}(\mathrm{mm} \mathrm{Hg})$ & $89(11)$ & $0.06(0.02,0.10)^{*}$ & $0.01(0.00,0.02)^{*}$ \\
\hline Physical activity (kJ/day) & $1,187(622-1,990)$ & $-0.03-0.07,0.01)$ & $-0.01(-0.01,0.00)$ \\
\hline \multicolumn{4}{|l|}{ Blood-based markers } \\
\hline Total cholesterol (mmol/L) & $5.90(1.08)$ & $0.11(0.07,0.15)^{* * *}$ & $0.02(0.01,0.03)^{* * *}$ \\
\hline
\end{tabular}

$\mathrm{BMI}$, body mass index; CHD, coronary heart disease; $\mathrm{Cl}$, confidence interval; $\mathrm{CRP}, \mathrm{C}$-reactive protein; DBP, diastolic blood pressure; HDL-C, high-density lipoprotein cholesterol; IQR, interquartile range; SD, standard deviation; SBP, systolic blood pressure; SES, socioeconomic status; Ref, reference. aPearson correlation coefficients between serum magnesium and the row variables. bercentage change in values of serum copper per 1 SD, increase in the row variable (or for categorical variables, the percentage difference in mean values of serum copper for the category vs. the reference). Asterisks indicate the level of statistical significance: $* p<0.05 ; * * p<0.01 ; * * p<0.001$.

ficients were calculated using linear regression models adjusted for age, to assess the cross-sectional associations of serum copper with various risk markers. We constructed Kaplan-Meier curves for tertiles of serum copper levels and compared them using the log rank test. Multivariable-adjusted hazard ratios (HRs) with 95\% confidence intervals (CIs) were calculated using Cox proportional hazard models, after confirming no major departure from the assumptions of proportionality of hazards using Schoenfeld residuals [24]. Serum copper was modeled as both continuous [per SD increase] and categorical (tertiles) variables for the estimation of the associations with both outcomes (VTE and CHD). HRs were calculated with adjustment for confounders in 3 progressive models: (model 1) age; (model 2) model 1 plus other established and emerging risk factors; and (model 3) model 2 plus other potential confounders. The confounders were selected based on their previously established role as risk factors for the outcomes assessed, evidence from previous research, or their potential as confounders based on known associations with the outcomes and observed associations with serum copper using the available data [25]. All statistical analyses were conducted using Stata version MP 16 (Stata Corp, College Station, TX, USA).

\section{Results}

The baseline characteristics of study participants and cross-sectional correlates of serum copper are presented in Table 1. The mean (SD) age and serum copper of the 2,492 men at baseline were 53 (5) years and $1.11(0.18)$ 
Table 2. Association between serum copper and risk of VTE

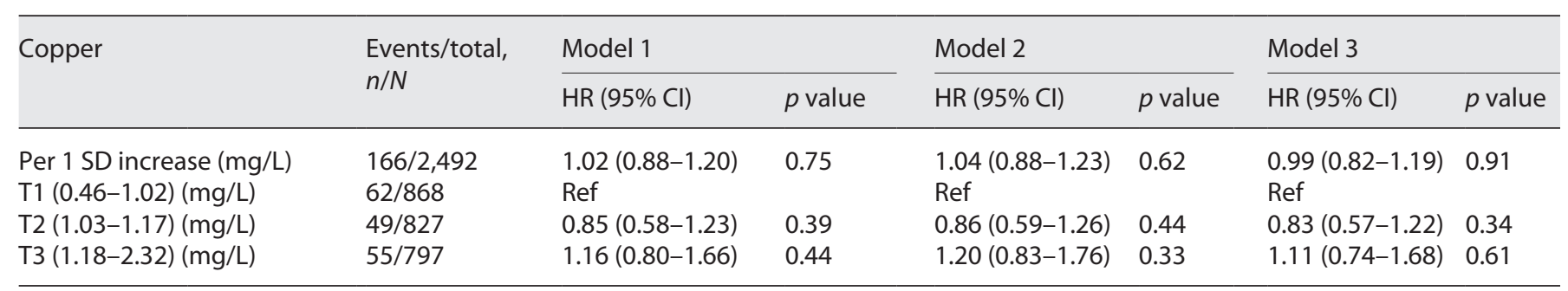

$\mathrm{Cl}$, confidence interval; HR, hazard ratio; Ref, reference; SD, standard deviation; T, tertile; VTE, venous thromboembolism; SES, socioeconomic status; BMI, body mass index; hsCRP, high-sensitivity C-reactive protein; CHD, coronary heart disease. Model 1: Adjusted for age. Model 2: Model 1 plus systolic blood pressure, BMI, total cholesterol, triglycerides, smoking status, history of type 2 diabetes, history of CHD, medication for dyslipidemia, alcohol consumption, physical activity, SES, and serum magnesium. Model 3: Model 2 plus hsCRP and history of cancer.

Fig. 1. Kaplan-Meier curves for coronary heart disease according to the tertiles of serum copper levels.

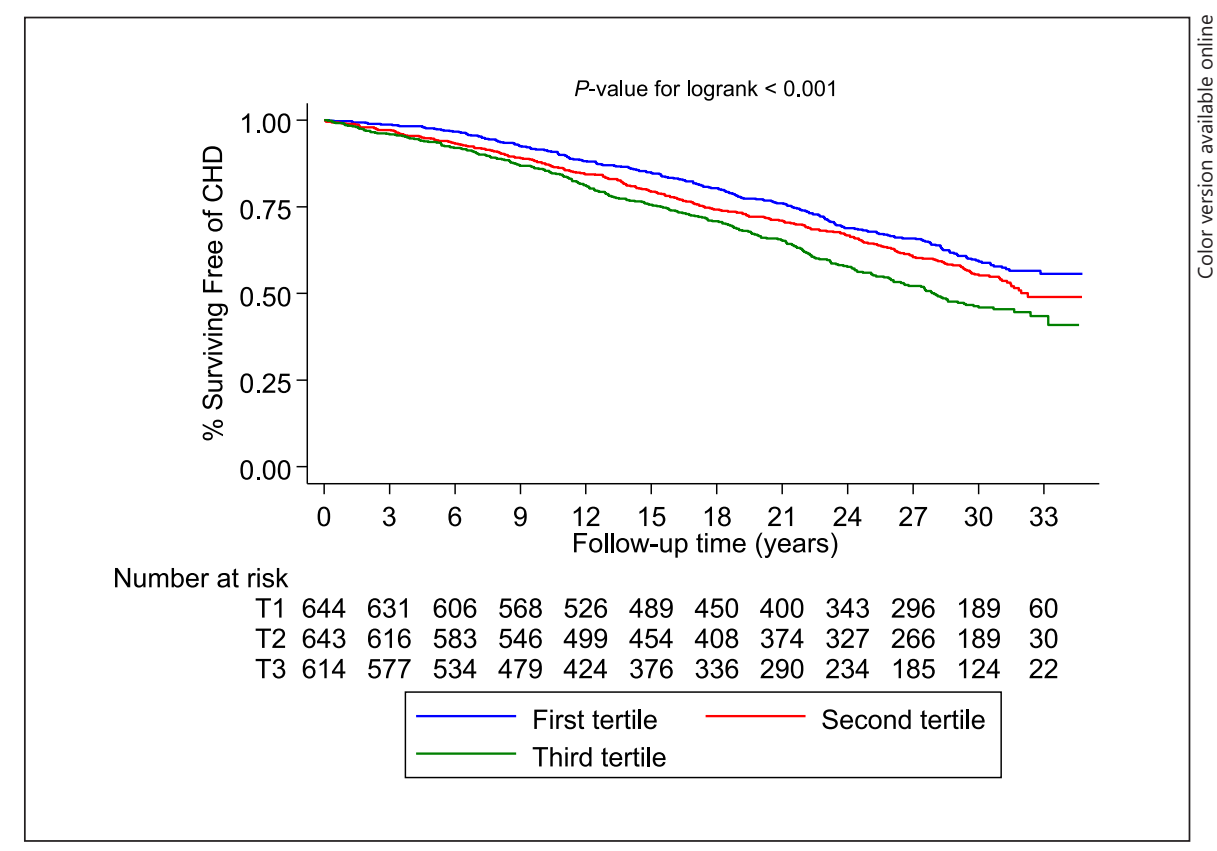

$\mathrm{mg} / \mathrm{L}$, respectively. Significant weak and positive correlations were observed between serum copper and age, alcohol consumption, socioeconomic status (SES), body mass index (BMI), blood pressure, and total cholesterol; serum copper was strongly and positively correlated with highsensitivity C-reactive protein (hsCRP) $(r=0.47)$. Significant weak and inverse correlations were observed with physical activity and serum magnesium. Values of serum copper were significantly higher in men who smoked and lower in those with a history of cancer.

During a median (IQR) follow-up of 27.0 (17.1-31.0) years, a total of 166 VTE cases corresponding to an an- nual rate of 2.83/1,000 person-years at risk (95\% CI: $2.43-$ $3.30)$ occurred. The HR (95\% CI) for VTE per 1 SD increase in serum copper in age-adjusted analysis was 1.02 (0.88-1.20), which became $1.04(0.88-1.23)$ on further adjustment for systolic blood pressure, BMI, total cholesterol, triglycerides, smoking status, histories of type 2 diabetes and CHD, medication for dyslipidemia, alcohol consumption, physical activity, SES, and serum magnesium. The HR (95\% CI) was attenuated on additional adjustment for hsCRP and history of cancer 0.99 (0.821.19) (Table 2). The corresponding adjusted HRs $(95 \%$ CIs) were $1.16(0.80-1.66), 1.20(0.83-1.76)$, and 1.11 
Table 3. Association between serum copper and risk of $\mathrm{CHD}$

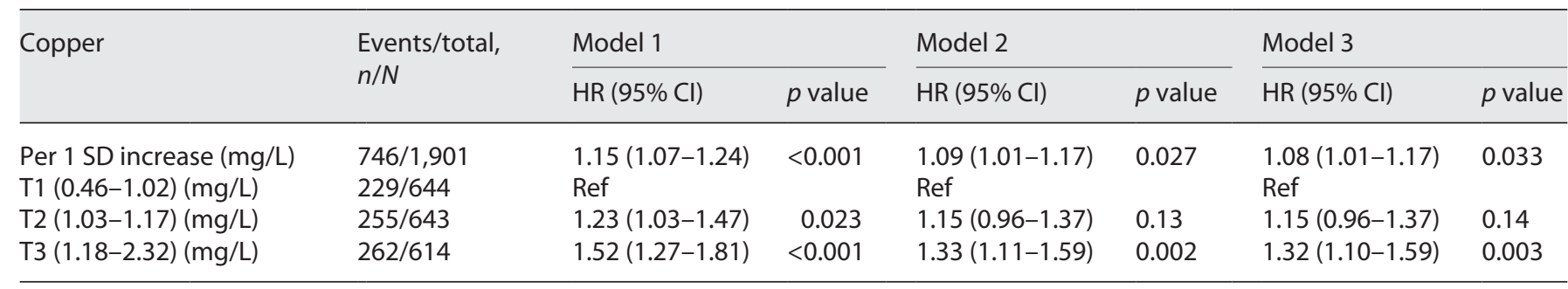

$\mathrm{Cl}$, confidence interval; $\mathrm{HR}$, hazard ratio; Ref, reference; $\mathrm{SD}$, standard deviation; $\mathrm{T}$, tertile; $\mathrm{CHD}$, coronary heart disease; $\mathrm{BMI}$, body mass index; BMI, body mass index; SBP, systolic blood pressure; HDL-C, high-density lipoprotein cholesterol. Model 1: Adjusted for age. Model 2: Model 1 plus SBP, BMI, total cholesterol, HDL-C, smoking status, and history of type 2 diabetes. Model 3: Model 2 plus alcohol consumption and physical activity.

(0.74-1.68), respectively, when comparing the top versus bottom tertiles of serum copper levels.

A total of $746 \mathrm{CHD}$ events were recorded during the follow-up period. Kaplan-Meier curves demonstrated a significant difference in the cumulative risk of $\mathrm{CHD}$ among males categorized by tertiles of serum copper levels $(p<0.001$ for log-rank test; Fig. 1). The HR (95\% CI) for CHD per $1 \mathrm{SD}$ increase in serum copper in analysis adjusted for several established risk factors was 1.09 (1.01-1.17), which remained consistent on further adjustment for alcohol consumption and physical activity 1.08 (1.01-1.17) (Table 3). The corresponding adjusted HRs (95\% CIs) were 1.33 (1.11-1.59) and 1.32 (1.101.59), respectively, when comparing the extreme tertiles of serum copper levels.

\section{Discussion}

Given existing evidence of a relationship between serum circulating copper and atherosclerotic CVD outcomes $[9,11-13]$, we sought to investigate the potential association between serum copper and VTE, an outcome that shares common risk factors $[2,3]$ and mechanistic pathways [4] with atherosclerotic CVD. In this general population-based cohort of middle-aged Caucasian men, we observed that increased serum levels of copper were not associated with the future risk of VTE. However, we demonstrated an association between elevated circulating copper levels and increased risk of $\mathrm{CHD}$, which is consistent with previous findings [11-13]. Given that this is the first population-based prospective study to evaluate the association of serum copper with future risk of VTE in a general population, these results are unchallenged or

Serum Copper, Atherosclerotic CVD, and VTE cannot be compared and will require future studies to confirm or refute these findings.

Copper is known to participate in cellular metabolism and functions as a cofactor in a number of cuproenzymes $[26,27]$, processes which are known to regulate cardiovascular function. Though copper is a major trace element which plays a beneficial role in numerous biological processes, it can exhibit toxic effects in excessive amounts. Mechanistic pathways proposed to link high serum levels of copper to increased risk of atherosclerotic CVD are via (i) oxidative modification of low-density lipoprotein cholesterol and free radical formation, which promote atherogenesis [28]; (ii) inflammation through the close relationship with ceruloplasmin, an acute phase reactant; (iii) insulin resistance and pathogenesis of diabetes [29], a major risk factor for $\mathrm{CHD}$; and (iv) luminal narrowing of the arteries, due to expansion of the arterial neointima caused by extracellular matrix molecules, whose major component is copper [30].

Given the strong link between atherosclerotic CVD and VTE and the wealth of evidence of a plausible relationship between serum copper and atherosclerotic CVD, our findings may be unexpected. However, though there is evidence to suggest that atherosclerotic CVD and VTE are closely related, they have historically been viewed as 2 distinct diseases [31]. It has been reported that atherosclerotic CVD is an underlying condition and precedes the development of VTE [32], whereas contrary evidence suggests atherosclerotic CVD does not precede VTE development $[33,34]$ or VTE rather precedes atherosclerotic disease [35]. Furthermore, findings on traditional risk factors for VTE and atherosclerotic CVD have not been consistent. Whereas some studies have demonstrated significant associations between traditional CVD risk 
factors and VTE risk $[3,36]$, others have not $[37,38]$. Hence, our null association between serum copper, an essential micronutrient, and VTE may suggest differences in the etiology and pathophysiology of arterial thrombotic disease and VTE. Other factors that could explain the lack of evidence of an association could be due to population and study design characteristics and/or limitations such as the age-group (middle-aged), male-only sex, low VTE event rate, and regression dilution bias due to availability of only baseline measurements of copper and the long follow-up duration. Regression dilution bias is known to underestimate the true strength of the association between an exposure and outcome, particularly for cohorts with long-term follow-up [39].

\section{Strengths and Limitations}

To our knowledge, our study is the first and largest of its kind to examine the relation of serum copper and VTE risk. Other strengths include the population-based prospective cohort design, the long-term and zero loss to follow-up, and adjustment for a comprehensive list of lifestyle factors and blood-based biomarkers. Other limitations include the possibility of residual confounding and lack of data on VTE subtypes. Furthermore, serum copper concentrations may not accurately reflect actual copper status; leukocyte copper measurement is considered a more reliable index of copper status in the body [9]. The generalizability of the findings to other populations and age-groups remains to be determined. Therefore, our results should be regarded as hypothesis generating. More studies are warranted to investigate the association and explore any potential biological mechanisms underlying the associations.

\section{Conclusions}

We have confirmed previously reported associations between high serum copper levels and increased risk of atherosclerotic CVD in a middle-aged male population, but serum copper was not associated with future VTE risk. Other large-scale prospective studies conducted in women, other age-groups, and other populations are needed to confirm or refute these findings.

\section{Acknowledgments}

The authors thank the staff of the Kuopio Research Institute of Exercise Medicine and the Research Institute of Public Health and University of Eastern Finland, Kuopio, Finland, for the data collection in the study.

\section{Statement of Ethics}

Written informed consent was obtained for each participant prior to baseline and follow-up data collections. This study was approved by the institutional review board of the University of Eastern Finland (reference \#:143/97) and complies with the Declaration of Helsinki.

\section{Conflict of Interest Statement}

The authors have no conflicts of interest to declare.

\section{Funding Sources}

Prof. Laukkanen acknowledges support from The Finnish Foundation for Cardiovascular Research, Helsinki, Finland. Dr. Kunutsor is funded by the NIHR Biomedical Research Centre at University Hospitals Bristol and Weston NHS Foundation Trust and the University of Bristol. The views expressed in this publication are those of the authors and not necessarily those of the NHS, the National Institute for Health Research, or the Department of Health and Social Care. These sources had no role in design and conduct of the study; collection, management, analysis, and interpretation of the data; and preparation, review, or approval of the manuscript.

\section{Author Contributions}

All authors contributed to conceptualization, methodology, and critical revision. S.K. Kunutsor conducted the statistical analysis and wrote the article.

\section{Data Availability Statement}

The data that support the findings of this study are available from the principal investigator (J.A.L.), upon reasonable request.

References

1 Barquera S, Pedroza-Tobías A, Medina C, Hernández-Barrera L, Bibbins-Domingo K, Lozano R, et al. Global overview of the epidemiology of atherosclerotic cardiovascular disease. Arch Med Res. 2015 Jul;46(5):328-38.

2 Glynn RJ, Rosner B. Comparison of risk factors for the competing risks of coronary heart disease, stroke, and venous thromboembolism. Am J Epidemiol. 2005 Nov 15;162(10): 975-82.

3 Ageno W, Becattini C, Brighton T, Selby R, Kamphuisen PW. Cardiovascular risk factors and venous thromboembolism: a meta-analysis. Circulation. 2008 Jan 1;117(1):93-102. 
4 Ray JG. Dyslipidemia, statins, and venous thromboembolism: a potential risk factor and a potential treatment. Curr Opin Pulm Med. 2003 Sep;9(5):378-84.

5 Cohen AT, Agnelli G, Anderson FA, Arcelus JI, Bergqvist D, Brecht JG, et al. Venous thromboembolism (VTE) in Europe. The number of VTE events and associated morbidity and mortality. Thromb Haemost. 2007 Oct;98(4):756-64.

6 Douketis JD, Gu CS, Schulman S, Ghirarduzzi A, Pengo V, Prandoni P. The risk for fatal pulmonary embolism after discontinuing anticoagulant therapy for venous thromboembolism. Ann Intern Med. 2007 Dec 4;147(11): 766-74.

7 Festa RA, Thiele DJ. Copper: an essential metal in biology. Curr Biol. 2011 Nov 8;21(21): R877-83.

8 Nath R. Copper deficiency and heart disease: molecular basis, recent advances and current concepts. Int J Biochem Cell Biol. 1997 Nov; 29(11):1245-54.

9 DiNicolantonio JJ, Mangan D, O’Keefe JH. Copper deficiency may be a leading cause of ischaemic heart disease. Open Heart. 2018; 5(2): 0000784.

10 Klevay LM. Ischemic heart disease as deficiency disease. Cell Mol Biol. 2004 Dec;50(8): 877-84.

11 Chen A, Li G, Liu Y. Association between copper levels and myocardial infarction: a meta-analysis. Inhal Toxicol. 2015;27(5): $237-46$.

12 Reunanen A, Knekt P, Aaran RK. Serum ceruloplasmin level and the risk of myocardial infarction and stroke. Am J Epidemiol. 1992 Nov 1;136(9):1082-90.

13 Ford ES. Serum copper concentration and coronary heart disease among US adults. Am J Epidemiol. 2000 Jun 15;151(12):1182-8.

14 Simera I, Moher D, Hoey J, Schulz KF, Altman DG. A catalogue of reporting guidelines for health research. Eur J Clin Invest. 2010 Jan;40(1):35-53.

15 Kunutsor SK, Laukkanen JA. Circulating active serum calcium reduces the risk of hypertension. Eur J Prev Cardiol. 2017 Feb;24(3): $239-43$.

16 Kunutsor SK, Whitehouse MR, Blom AW, Laukkanen JA. Low serum magnesium levels are associated with increased risk of fractures: a long-term prospective cohort study. Eur J Epidemiol. 2017 Jul;32(7):593-603.

17 Kunutsor SK, Seidu S, Blom AW, Khunti K, JAL. Serum C-reactive protein increases the risk of venous thromboembolism: a prospective study and meta-analysis of published prospective evidence. Eur J Epidemiol. 2017; 32(8):657-67.

18 Kunutsor SK, Seidu S, Katechia DT, Laukkanen JA. Inverse association between serum albumin and future risk of venous thromboembolism: interrelationship with high sensitivity C-reactive protein. Ann med. $2018 \mathrm{Feb}$ 20;50(3):240-8.

19 Kunutsor SK, Makikallio TH, Araujo CGS, Jae SY, Kurl S, Laukkanen JA. Cardiorespiratory fitness is not associated with risk of venous thromboembolism: a cohort study. Scand Cardiovasc J. 2019 Oct;53(5):255-8.

20 Kunutsor SK, Makikallio TH, Khan H, Laukkanen T, Kauhanen J, Laukkanen JA. Sauna bathing reduces the risk of venous thromboembolism: a prospective cohort study. Eur J Epidemiol. 2019 Oct;34(10):983-6.

21 Kunutsor SK, Makikallio TH, Voutilainen A, Laukkanen JA. Handgrip strength is not associated with risk of venous thromboembolism: a prospective cohort study. Scand Cardiovasc J. 2020 Apr 11;54(4):253-7.

22 Kunutsor SK, Laukkanen JA. Circulating serum magnesium and the risk of venous thromboembolism in men: a Long-Term Prospective Cohort Study. Pulse. 2021 Jun;8(34):108-13.

23 Laukkanen JA, Kurl S, Lakka TA, Tuomainen TP, Rauramaa R, Salonen R, et al. Exerciseinduced silent myocardial ischemia and coronary morbidity and mortality in middleaged men. J Am Coll Cardiol. 2001 Jul;38(1): 72-9.

24 Therneau TM, Grambsch PM. Modeling Survival Data: Extending the Cox Model. New York, NY: Springer; 2000.

25 Groenwold RH, Klungel OH, Grobbee DE, Hoes AW. Selection of confounding variables should not be based on observed associations with exposure. Eur J Epidemiol. 2011 Aug; 26(8):589-93.

26 Linder MC, Hazegh-Azam M. Copper biochemistry and molecular biology. Am J Clin Nutr. 1996 May;63(5):797S-811.

27 Camakaris J, Voskoboinik I, Mercer JF. Molecular mechanisms of copper homeostasis. Biochem Biophys Res Commun. 1999 Aug 2; 261(2):225-32.

28 Heinecke JW, Rosen H, Chait A. Iron and copper promote modification of low density lipoprotein by human arterial smooth muscle cells in culture. J Clin Invest. 1984 Nov;74(5): $1890-4$.
29 Tanaka A, Kaneto H, Miyatsuka T, Yamamoto K, Yoshiuchi K, Yamasaki Y, et al. Role of copper ion in the pathogenesis of type 2 diabetes. Endocr J. 2009;56(5):699-706.

30 Ferns GA, Lamb DJ, Taylor A. The possible role of copper ions in atherogenesis: the Blue Janus. Atherosclerosis. 1997 Sep;133(2):139-52.

31 Prandoni P. Venous thromboembolism and atherosclerosis: is there a link? J Thromb Haemost. 2007 Jul;5(Suppl 1):270-5.

32 Prandoni P, Bilora F, Marchiori A, Bernardi E, Petrobelli F, Lensing AW, et al. An association between atherosclerosis and venous thrombosis. N Engl J Med. 2003 Apr 10; 348(15):1435-41.

33 Reich LM, Folsom AR, Key NS, Boland LL, Heckbert SR, Rosamond WD, et al. Prospective study of subclinical atherosclerosis as a risk factor for venous thromboembolism. J Thromb Haemost. 2006 Sep;4(9):1909-13.

34 van der Hagen PB, Folsom AR, Jenny NS, Heckbert SR, O'Meara ES, Reich LM, et al. Subclinical atherosclerosis and the risk of future venous thrombosis in the Cardiovascular Health Study. J Thromb Haemost. 2006 Sep; 4(9):1903-8.

35 Prandoni P, Ghirarduzzi A, Prins MH, Pengo V, Davidson BL, Sorensen H, et al. Venous thromboembolism and the risk of subsequent symptomatic atherosclerosis. J Thromb Haemost. 2006 Sep;4(9):1891-6.

36 Gregson J, Kaptoge S, Bolton T, Pennells L, Willeit P, Burgess $S$, et al. Cardiovascular risk factors associated with venous thromboembolism. JAMA Cardiol. 2019 Feb 1;4(2):16373.

37 Wattanakit K, Lutsey PL, Bell EJ, Gornik H, Cushman M, Heckbert SR, et al. Association between cardiovascular disease risk factors and occurrence of venous thromboembolism. A time-dependent analysis. Thromb Haemost. 2012 Sep;108(3):508-15.

38 Mahmoodi BK, Cushman M, Anne Naess I, Allison MA, Bos WJ, Braekkan SK, et al. Association of traditional cardiovascular risk factors with venous thromboembolism: an individual participant data meta-analysis of prospective studies. Circulation. 2017 Jan 3; 135(1):7-16.

39 MacMahon S, Peto R, Cutler J, Collins R, Sorlie $\mathrm{P}$, Neaton J, et al. Blood pressure, stroke, and coronary heart disease. Part 1, prolonged differences in blood pressure: prospective observational studies corrected for the regression dilution bias. Lancet. 1990 Mar 31; 335(8692):765-74. 